\section{PERCOLATION THEORY}

Live transmission

Cell Syst. 7, 137-145 (2018)

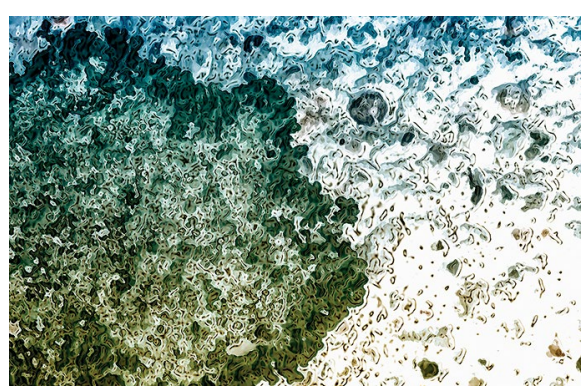

Credit: GiroScience/Alamy Stock Photo

When a bacterium in the middle of a biofilm feels starved of nutrients, it releases a potassium signal that impacts nutrient uptake in its neighbours, starting a chain reaction that propagates all the way to the edge of the film. The peripheral cells respond by reducing consumption so the interior cells have access to nutrients. This display of altruism incurs a high cost to the individual - and cell-to-cell heterogeneity makes it a nontrivial problem in signal transmission. Now, Joseph Larkin and colleagues have shown that the benefit outweighs the individual cost when the system is poised near a percolation threshold.

The authors' model predicts a powerlaw decay in the distribution of cluster sizes and puts the fraction of cells required for signalling connectivity at a little less than half. By incorporating excitable dynamics into their framework, they found a minimum in the cost-benefit ratio near the percolation threshold. Experiments placed Bacillus subtilis biofilms near this threshold, whereas mutant strands with modified structure and dynamics strayed away, suggesting the biofilm's spatial heterogeneity is tuned to ensure efficient transmission.

https://doi.org/10.1038/s41567-018-0321-7

\section{TOPOLOGICAL OPTICS}

\section{Light on skyrmions}

Science 361, 993-996 (2018)

Best thought of as topological excitations that share the same topology as a set of magnetic moments wrapped around a sphere, skyrmions are widely thought to be promising for magnetic storage and spintronics applications. Shai Tsesses and colleagues have now brought skyrmions into the arena of optics. By controlling the interference of plasmon polaritons on a patterned metallic surface, they have succeeded in creating a lattice of optical skyrmions. The authors were able to image this pattern of evanescent electromagnetic fields using a phase-resolved near-field optical microscope.

In contrast to their magnetic analogues studied in solid-state systems, these optical skyrmions can be continuously tuned from a so-called bubble- to Néel-type structure. However, in common with many such topological excitations, the lattice of optical skyrmions displays a remarkable robustness to imperfections. The extent to which light can be manipulated and processed in photonic systems has already given us countess technologies. Few would bet against a few more popping out following this discovery of photonic skyrmions.

https://doi.org/10.1038/s41567-018-0322-6

\section{D MATERIALS}

\section{Mapping the moiré \\ 2D Mater. 5, 041008 (2018)}

Understanding the nanoscale structure of materials has always been an important problem, but recent observations of superconductivity in twisted bilayer graphene with a moiré pattern make it particularly urgent for this material. A substantial advance has been made by Oleg Ovchinnikov and collaborators by combining very sensitive electron microscopy techniques with computational modelling to obtain a threedimensional map of the crystal lattice.

Crucially, they were able to determine not only the lateral position of all the atoms from the electron microscopy, but also the height of the ripples from density functional theory calculations. This complete characterization of the material means that local relaxation induced by the bilayer moiré pattern can be distinguished from large-scale strain effects. The former may be vital in understanding the superconductivity, while the latter are important because they have a detrimental effect on conventional transport properties.

https://doi.org/10.1038/s41567-018-0323-5

\section{QUANTUM COMPUTING}

\section{Let's get practical}

Proc. Natl Acad. Sci. USA https://doi.org/ctvz (2018)

We keep hearing about the tough problems that quantum computers will allow us to crack. As tantalizing - and potentially useful - as they sound, our experimental capabilities still relegate them to a distant future. Many have thus turned towards the more immediate goal of finding tasks beyond the reach of classical machines, but the utility of these tasks is rarely a priority. Andrew Childs and colleagues have now taken a more practical look at what lies between these extremes.

Through circuit optimization, Childs et al. characterized truly useful computations whose hardware requirements are not too far out of reach. They specifically considered the full quantum dynamics of $1 \mathrm{D}$ spin chains - a notoriously difficult system to simulate for any more than 20 or so spins, which is often too few to fully reveal phase transitions. The authors showed that digital quantum simulation of a 50 -spin chain could be achieved by current algorithms with fewer than 100 qubits executing around $10^{8}$ gates - orders of magnitude below the number needed for factoring large numbers or simulating quantum chemistry.

https://doi.org/10.1038/s41567-018-0324-4 Stefanie Reichert and Andrea Taroni 